Review began 12/21/2021 Review ended 02/18/2022 Published 02/22/2022

(๑) Copyright 2022

Saxena et al. This is an open access article distributed under the terms of the Creative Commons Attribution License CC-BY 4.0. which permits unrestricted use, distribution, and reproduction in any medium, provided the original author and source are credited.

\section{Strength, Weakness, Opportunities, and Threats (SWOT) Analysis of Virtual Outpatient Department Under Telemedicine Department During the COVID-19 Pandemic}

Vartika Saxena ${ }^{1}$, Yogesh Bahurupi ${ }^{1}$, Ashutosh Mishra ${ }^{1}$, Ashok Singh ${ }^{2}$, Shailesh Parate ${ }^{3}$, Harindra Sandhu 4

1. Department of Community and Family Medicine, All India Institute of Medical Sciences, Rishikesh, Rishikesh, IND 2 Department of Pathology, All India Institute of Medical Sciences, Rishikesh, Rishikesh, IND 3. Department of Forensic Medicine, All India Institute of Medical Sciences, Rishikesh, Rishikesh, IND 4. Department of General Surgery, Lala Lajpat Rai Memorial Medical College, Meerut, IND

Corresponding author: Ashutosh Mishra, ashutoshdr23@gmail.com

\section{Abstract}

\section{Background}

COVID-19 pandemic has challenged all current management tools used for patient care. This study aims to determine strength, weakness, opportunities and threats (SWOT) to virtual OPD and consultants' perceptions of teleconsultation virtual OPD during the COVID-19 pandemic, adapting to newer technologies for successfully handling this situation.

\section{Material and methods}

A facility-based cross-sectional study was conducted at the Virtual OPD of All India Institute of Medical Sciences Rishikesh among patients availing Telemedicine consultation during the COVID-19 pandemic. All patients availing services from April 2020 to October 2020 were included in this study. Method for SWOT analysis: A checklist was prepared, and investigators assessed SWOT. An external evaluator was invited to evaluate the SWOT analysis conducted by the investigators. For numeric variables, the mean \pm SD was used, and for categorical variables, percentages and proportions were used.

\section{Results}

Around $22 \%$ of the patients who approached virtual OPDs were $\geqslant 60$ years of age. More than half $(55.3 \%)$ of the patients or their attendants who consulted Telemedicine OPD were from Uttarakhand, followed by Uttar Pradesh (42.6\%). The proportions of male patients were $54.4 \%$. Around $17.6 \%$ of teleconsultations were performed for radiotherapy. General medicine and cardiology consultations were (15.2\%) and (12.8\%), respectively.

\section{Conclusion}

Telemedicine can be effective if certain requirements had been provided. Maintaining privacy of the patient's data was a challenge.

Categories: Healthcare Technology, Other, Epidemiology/Public Health

Keywords: threats, opportunities, weakness, strength, swot analysis, virtual opd, telemedicine, covid-19

\section{Introduction}

Telemedicine can be defined as the delivery of healthcare services, where distance is a critical factor, by all healthcare professionals using information and communication technologies for the exchange of valid information for the diagnosis, treatment, and prevention of disease and injuries, for research and evaluation, and for the continuing education of healthcare providers, all in the interests of advancing the health of individuals and their communities [1].

Due to the COVID-19 pandemic, telemedicine services were better options for patients to receive overall treatment from a distance and stay safely at home during and after the lockdown period.

Telemedicine aims to ensure equitable offerings to everyone, are cost-effective, offer protection to sufferers and medical doctors for the duration of pandemics, and provide timely and quicker care [2].

The non-accessibility of technology and communications is a key barrier in telemedicine in developing 
countries. According to the International Telecommunications Union data, only $31 \%$ of the developing world population uses the internet, of which $16 \%$ is used in Africa, and $90 \%$ of households do not have access to the Internet in the developing world [3].

The COVID-19 virus has created a pandemic and has challenged all current management tools used for patient care. The challenges this pandemic created are huge and have created new avenues for handling the situation. The challenge we face is providing healthcare facilities without coming into close contact with patients.

This study aims to determine "strength, weakness, opportunities, and threats (SWOT)" to the virtual outpatient department (OPD) and consultants' perceptions of teleconsultation virtual OPD during the COVID-19 pandemic, adapting to newer technologies for successfully handling this situation. We also highlight the difficulties faced in starting up services with limited resources. This study focused on patients availing teleconsultation services through virtual OPD and consultants involved in virtual OPD at All India Institute of Medical Sciences (AIIMS) Rishikesh during the COVID-19 pandemic. The purpose of this study was to assess the SWOT analysis and profile of patients who visited the AIIMS Rishikesh virtual OPD via telemedicine.

\section{Materials And Methods}

A facility-based cross-sectional study was conducted at the virtual OPD of AIIMS Rishikesh among patients availing telemedicine consultation services at AIIMS Rishikesh during the COVID-19 pandemic. All patients availing services from April to October 2020 (seven months) were included in this study. Records of patients availing teleconsultation services were accessed for their profiles. Incomplete records were excluded from the final analyses.

\section{Method for SWOT analysis}

A checklist was prepared after review of relevant literature on telemedicine practices, which included facilitating and hindering factors on telemedicine. An external evaluator was invited to conduct the SWOT analysis by the investigators.

\section{Criteria used for external evaluators}

External evaluators have worked in telemedicine/telehealth with a public health background. An invitation was sent and a checklist prepared for SWOT analysis was vetted for appropriateness and suggestions.

All the data collected during the teleconsultation period provided by patients through telephone, WhatsApp, and video calls were evaluated and reviewed.

\section{Data analysis}

Microsoft Excel 2010 was used for entering the data. EPI Info 7.0, a professional statistical tool for Windows, was used to analyze the data. For numeric variables, the mean \pm SD was used, and for categorical variables, percentages and proportions were used.

\section{Results}

In this study, a total of 5,278 patients availed teleconsultation service from virtual OPD of AIIMS Rishikesh during the COVID-19 pandemic between April 20, 2020, and October 31, 2020.

Around $22 \%$ of the patients who approached virtual OPDs were $\geqslant 60$ years of age, and $12 \%$ of the patients were between 46 and 50 years of age. Approximately $9.6 \%$ and $9.5 \%$ of the patients were between 36 to 40 years of age and 41 to 45 years of age, respectively. The percentage of patients under five years of age was $3.8 \%$. The proportion of males was $54.4 \%$ compared with female patients (45.6\%) in the telemedicine OPD (Table 1). 


\section{Cureus}

\begin{tabular}{|c|c|c|}
\hline Variable & Categories & Number (\%) \\
\hline \multirow{13}{*}{ Age (in years) } & 0 to 5 & $201(3.8 \%)$ \\
\hline & 6 to 10 & $88(1.7 \%)$ \\
\hline & 11 to 15 & $105(2 \%)$ \\
\hline & 16 to 20 & 168 (3.2\%) \\
\hline & 21 to 25 & 361 (6.8\%) \\
\hline & 26 to 30 & $398(7.5 \%)$ \\
\hline & 31 to 35 & $430(8.2 \%)$ \\
\hline & 36 to 40 & $508(9.6 \%)$ \\
\hline & 41 to 45 & $502(9.5 \%)$ \\
\hline & 46 to 50 & $633(12 \%)$ \\
\hline & 51 to 55 & $476(9.1 \%)$ \\
\hline & 56 to 59 & $245(4.6 \%)$ \\
\hline & $\geq 60$ & $1163(22 \%)$ \\
\hline \multirow{2}{*}{ Gender } & Male & $2870(54.4 \%)$ \\
\hline & Female & 2408 (45.6\%) \\
\hline
\end{tabular}

TABLE 1: Age- and genderwise distribution of patients availing teleconsultation at the virtual OPD $(\mathrm{N}=5,278)$

OPD, outpatient department

More than half (55.3\%) of the patients or their attendants who consulted telemedicine OPD were from Uttarakhand. Approximately $42.6 \%$ of the patients or their attendants were from Uttar Pradesh. Only $2.1 \%$ of the patients who approached telemedicine OPD were from other states of India (Table 2).

\begin{tabular}{|l|c|}
\hline State & Number (\%) \\
\hline Uttarakhand & $2918(55.3 \%)$ \\
\hline Uttar Pradesh & $2246(42.6 \%)$ \\
Others & $114(2.1 \%)$ \\
\hline
\end{tabular}

TABLE 2: Geographical distribution of patients availing teleconsultation at the virtual OPD $(\mathrm{N}=5,278)$

OPD, outpatient department

Around $17.6 \%$ of the teleconsultations were conducted for radiotherapy. General medicine and cardiology consultations were $15.2 \%$ and $12.8 \%$, respectively. Approximately $7.7 \%$ of the calls of patients were required for orthopedic consultation. For Chemotherapy, General Surgery, Pediatrics, and obstetrics and gynecology (OBG), 6.5\%, 5.1\%, 4.5\%, and 3.7\% of the calls were attended, respectively (Table 3). 


\section{Cureus}

\begin{tabular}{|c|c|}
\hline Clinical specialties & Number (\%) \\
\hline General medicine & $801(15.2 \%)$ \\
\hline General surgery & $271(5.1 \%)$ \\
\hline Orthopedics & 407 (7.7\%) \\
\hline Pediatrics & 240 (4.5\%) \\
\hline Obstetrics and gynecology & $196(3.7 \%)$ \\
\hline Dermatology & $77(1.5 \%)$ \\
\hline Psychiatry & $93(1.8 \%)$ \\
\hline Radiology & $10(0.2 \%)$ \\
\hline Ophthalmology & $71(1.3 \%)$ \\
\hline ENT & $93(1.8 \%)$ \\
\hline Community and family medicine & $134(2.5 \%)$ \\
\hline Hematology & $22(0.4 \%)$ \\
\hline Medical oncology & $345(6.5 \%)$ \\
\hline Surgical oncology & $64(1.2 \%)$ \\
\hline Gastroenterology & $103(2 \%)$ \\
\hline Plastic surgery & $20(0.4 \%)$ \\
\hline Neurosurgery & $61(1.2 \%)$ \\
\hline Neurology & $123(2.3 \%)$ \\
\hline Radiotherapy & $930(17.6 \%)$ \\
\hline Urology & $209(4 \%)$ \\
\hline Nephrology & 69 (1.3\%) \\
\hline Emergency medicine & $28(0.5 \%)$ \\
\hline AYUSH & $3(0.1 \%)$ \\
\hline Pathology & $13(0.2 \%)$ \\
\hline Cardiology & $676(12.8 \%)$ \\
\hline Pediatric surgery & $8(0.2 \%)$ \\
\hline Pulmonary medicine & $74(1.4 \%)$ \\
\hline Hemato-oncology & $26(0.5 \%)$ \\
\hline PMR & $2(0.0 \%)$ \\
\hline CTVS & $46(0.9 \%)$ \\
\hline Breast clinic & $50(1 \%)$ \\
\hline Dentistry & $13(0.2 \%)$ \\
\hline
\end{tabular}

TABLE 3: Distribution of patients availing teleconsultation at the virtual OPD according to various specialties $(\mathrm{N}=5,278)$

CTVS, cardiothoracic and vascular surgery; OPD, outpatient department; PMR, physical medicine and rehabilitation

Nearly three-fourths (68.7\%) of the patients were counselled during telephonic consultation. Approximately $12.7 \%$ of the patients or their relatives were counselled, and medicines were prescribed. Medications were 


\section{Cureus}

prescribed to $5 \%$ of the patients. A total of $11.1 \%$ of the patients were advised to undergo investigations. Only a very few patients $(0.6 \%)$ were referred (Table 4$)$.

\begin{tabular}{|l|c|}
\hline Variable & Number (\%) \\
\hline Counselling & $3626(68.7 \%)$ \\
\hline Medicine prescribed & $264(5 \%)$ \\
Both counselling and medicines & $672(12.7 \%)$ \\
\hline Investigation advised & $588(11.1 \%)$ \\
Counselling, medicine, and investigations advised & $99(1.9 \%)$ \\
\hline Counselling and referred & $29(0.6 \%)$ \\
\hline
\end{tabular}

TABLE 4: Details of action taken by the attending doctors for the patients availing teleconsultation at the virtual OPD $(\mathrm{N}=5,278)$

OPD, outpatient department

\section{SWOT analysis of telemedicine consultation \\ Strengths}

- The patients require no prior registration, and the consent is considered implied.

- Considering the pandemic, no physical contact with patients minimizes the risk of exposure as consultation is through telecommunication.

- There is reduced out-of-pocket expenditure as traveling and other expenses are excluded.

- Human resources are better utilized.

- Accessibility of primary and tertiary healthcare through Teleconsultation is made to all.

- Patients are comfortable with Teleconsultation with the present digitalized trends.

- Minimum infrastructure is required to practice telemedicine.

\section{Weaknesses}

- Teleconsultation quality depends on the speed and connectivity of internet services.

- Minimal emergency services are provided by telemedicine consultation.

- For cases that require patient counselling or where there is a need for "breaking bad news," telemedicine consultation is inappropriate.

- There is poor quality of report exchange.

Services of telemedicine are inaccessible to patients who are illiterate or do not have access to technology.

Often calls are made only for inquiry purposes rather than for consultation

- Arranging referral and transport services for interstate patients is difficult.

The quality of patient data achieved is poor.

- There is an absence of physical examination of patients.

- There is a shortage of trained staff. 
- The same doctor may not be available for follow-up.

\section{Opportunities}

- The referral mechanism can be strengthened through telemedicine.

- For better patient diagnosis, point-of-care technology can be utilized through the hub-and-spoke model.

- Patient's records can be digitalized through the creation of Electronic Health Record (EHR) and linking them to their Unique Health Identification Number (UHID).

- Geolocation of the patient can be used for an early investigation of a potential outbreak.

- Stringent guidelines should be proposed to avoid medicolegal issues and violation of privacy.

\section{Threats}

- Record keeping is a major lacuna of teleconsultation, which may lead to medicolegal issues.

- The same physician may not be available for every teleconsultation.

- Privacy of the patient, especially vulnerable groups, while communicating with a doctor can be hampered.

- Poor quality of telecommunication due to inadequate internet speed or network issues may lead to poor quality patient care.

- Lack of awareness among beneficiaries regarding services being provided through telemedicine.

\section{Discussion}

Sharing of symptoms and eliciting signs is crucial for the diagnosis of any health condition. There are a number of technologies that can be used in telemedicine, which can help patients adhere better to their medication regimens and manage their diseases better [2].

Alharbi et al. found that $54 \%$ of the patients were male and the remaining $46 \%$ were female [4]. Polinski et al. reported $70 \%$ of female patients in their study [5]. In comparison to this, in the present study, the proportion of males was $54.4 \%$ compared with female patients (45.6\%) in the telemedicine OPD. This finding might be due to the many patients included in this study, different sex ratios of the population, and a study conducted for a longer duration. In this study, around $17.6 \%$ of the teleconsultations were performed for Radiotherapy. General medicine and cardiology consultations were $15 \%$ and $12.7 \%$, respectively. Approximately $7.6 \%$ and $6.5 \%$ of patients' calls were for orthopedic consultation and chemotherapy, respectively. In contrast, Alharbi et al. in their study found that most (58.1\%) of the participants attended a virtual clinic of family medicine and the dermatology and pediatrics virtual clinics were attended by $15 \% 11.8 \%$ of the participants, respectively [4].

Telemedicine offers a variety of healthcare services by the healthcare providers, especially by the doctors, through the virtual clinic. In our study, the modes of communication were video, audio, and text messages. Telemedicine was instrumental during the pandemic when the lockdown was enforced worldwide. In Paris, among all the general practitioners, approximately $44 \%$ of them attended at least one teleconsultation during a pandemic [6]. With the improvement in communication technologies, telemedicine depicts a succession of medicine, and it should be established in all urban medical centers [7]. During the COVID-19 pandemic, virtual OPDs of AIIMS Rishikesh provided healthcare facilities to thousands of patients from all over India, especially during the lockdown period when patients had no option other than virtual consultation with the doctors/consultants. Internet-based audio and video services were also offered through WhatsApp.

Telemedicine is cost-effective because of the falling cost of internet services and cheaper devices [8]. During the COVID-19 pandemic, telemedicine services act as a barrier to the spread of COVID infection [9]. Telemedicine comes up with 4Cs: “care, convenience, comfort, and confidentiality" [10,11]. Telemedicine services save the important time and money of those patients who are critically ill and need urgent emergency care. Triage could be faster and more efficient in an emergency by implementing telemedicine in emergency care and helps in improvements in waiting time and patient satisfaction [12].

During the lockdown period in India, when there was no opportunity for a face-to-face consultation with the doctor for chronic diseases, and most patients benefitted from telemedicine at AIIMS Rishikesh.

Telemedicine was a useful tool to manage patients with diabetes during the lockdown period [13]. This study was conducted to assess the SWOT and profile of patients attending virtual OPD under telemedicine at AIIMS Rishikesh. Telemedicine's main benefit is that it allows patients to communicate with doctors while 
at home and thus helps the patients avoid the risk of getting the infection by coronavirus as avoiding the visit to the hospital for the treatment [14]. The disadvantage of telemedicine could be the clinical diagnosis errors because of the lack of examination of the patients. The diagnosis is completely dependent on the patients' history and/or their investigation reports, which are also difficult to obtain during the lockdown period due to the COVID-19 pandemic.

Professionalism should be maintained throughout the teleconsultation/virtual meeting with patients by using a high-resolution camera and seeing their face in the video. There is a need for a high-quality Wi-Fi or network signal [15].

In this study, around $22 \%$ of the patients who approached virtual OPD were $\geqslant 60$ years of age. In comparison, Alharbi et al. in their study including 439 respondents found that $12.3 \%$ of the patients were $\geqslant 60$ years of age [4]. This finding might be due to the demographic composition of the population, and patients more than 60 years of age had a higher chance of getting a severe infection by COVID-19, and travel is also risky during the COVID-19 pandemic. Consultation utilizing Telemedicine was more favored by the patients aged 68 years (range: $58-75$ years) than patients aged 76 years (range: $70-79.2$ years) during the COVID-19 pandemic [16]. During the pandemic, telemedicine proved to be a reliable technique not only for patient consultation but also for follow-up, as clinically unnecessary visits may increase COVID-19 exposure and infectious risk for patients and providers [17].

In the present study, more than half (55.3\%) of the patients or their attendants who consulted telemedicine OPD were from Uttarakhand. Approximately $42.6 \%$ and $2.1 \%$ of the patients or their attendants who approached telemedicine OPD were from Uttar Pradesh and other states of India, respectively. In this study, around two-thirds (68.7\%) of the patients were counselled during telephonic consultation. Approximately $12.7 \%$ of the patients or their relatives were counselled, and medicines were prescribed. Medications were prescribed to $5 \%$ of patients. A total of $11.1 \%$ of the patients were advised to undergo investigations. Telemedicine has become a critical tool for protecting access to care and responding to public health demands as a result of the COVID-19 pandemic. However, it has stressed the importance of addressing concerns about a lack of access to technology and high-speed internet, as well as inadequate digital literacy, which may hinder some populations from obtaining virtual treatment [18].

\section{Conclusions}

In this study, we performed SWOT analysis of telemedicine consultations. Telemedicine can be effective if certain requirements had been provided. Record keeping is a major lacuna of teleconsultation. Around 22\% of the patients who approached telemedicine OPD were $\geqslant 60$ years of age. The proportion of male patients was higher than that of female. More than half of the patients or their attendants who consulted telemedicine OPD were from Uttarakhand. Maximum consultations were performed for radiotherapy, and most of the patients were counselled during teleconsultation.

\section{Additional Information \\ Disclosures}

Human subjects: Consent was obtained or waived by all participants in this study. Institutional Ethics Committee, All India Institute of Medical Sciences, Rishikesh issued approval 370/IEC/IM/NF/2020. This is in reference to (No. 370/IEC/IM/NF/2020) your research proposal titled "SWOT Analysis of virtual OPD under Telemedicine department during COVID-19 Pandemic", Institutional Ethics Committee, All India Institute of Medical Sciences, Rishikesh has approved this research proposal in its present form. Animal subjects: All authors have confirmed that this study did not involve animal subjects or tissue. Conflicts of interest: In compliance with the ICMJE uniform disclosure form, all authors declare the following: Payment/services info: All authors have declared that no financial support was received from any organization for the submitted work. Financial relationships: All authors have declared that they have no financial relationships at present or within the previous three years with any organizations that might have an interest in the submitted work. Other relationships: All authors have declared that there are no other relationships or activities that could appear to have influenced the submitted work.

\section{References}

1. World Health Organization. A health telematics policy in support of WHO's Health-For-All strategy for global health development. (1997). Accessed: February 1, 2022:

https://apps.who.int/iris/bitstream/handle/10665/63857/WHO_DGO_98.1.pdf?sequence=1 \&isAllowed=y.

2. Telemedicine Practice Guidelines. (2020). Accessed: November 6, 2021: https://www.mohfw.gov.in/pdf/Telemedicine.pdf.

3. After Access 2018: A Demand-Side View of Mobile Internet from 10 African Countries . (2019). Accessed: February 1, 2022: https://www.africaportal.org/publications/after-access-2018-demand-side-view-mobileinternet-10-african-countries/.

4. Alharbi KG, Aldosari MN, Alhassan AM, Alshallal KA, Altamimi AM, Altulaihi BA: Patient satisfaction with virtual clinic during coronavirus disease (COVID-19) pandemic in primary healthcare, Riyadh, Saudi Arabia. J Family Community Med. 2021, 28:48-54. 10.4103/jfcm.JFCM_353_20 
5. Polinski JM, Barker T, Gagliano N, Sussman A, Brennan TA, Shrank WH: Patients' satisfaction with and preference for telehealth visits. J Gen Intern Med. 2016, 31:269-75. 10.1007/s11606-015-3489-X

6. Ohannessian R, Duong TA, Odone A: Global telemedicine implementation and integration within health systems to fight the COVID-19 pandemic: a call to action. JMIR Public Health Surveill. 2020, 6:e18810. $10.2196 / 18810$

7. Clark PA, Capuzzi K, Harrison J: Telemedicine: medical, legal and ethical perspectives. Medical Science Monitor. 2010, 30:261-72.

8. de la Torre-Díez I, López-Coronado M, Vaca C, Aguado JS, de Castro C: Cost-utility and cost-effectiveness studies of telemedicine, electronic, and mobile health systems in the literature: a systematic review. Telemed J E Health. 2015, 21:81-5. 10.1089/tmj.2014.0053

9. Rockwell KL, Gilroy AS: Incorporating telemedicine as part of COVID-19 outbreak response systems . Am J Manag Care. 20201, 26:147-8.

10. Beck CA, Beran DB, Biglan KM, et al.: National randomized controlled trial of virtual house calls for Parkinson disease. Neurology. 2017, 89:1152-61. 10.1212/WNL.0000000000004357

11. Bloem BR, Dorsey ER, Okun MS: The coronavirus disease 2019 crisis as Catalyst for telemedicine for chronic neurological disorders. JAMA Neurol. 2020, 77:927-8. 10.1001/jamaneurol.2020.1452

12. Aungst LA: Can telemedicine improve triage and patient satisfaction in urgent care settings? J Am Assoc Nurse Pract. 2019, 31:162-6. 10.1097/JXX.0000000000000117

13. Ghosh A, Gupta R, Misra A: Telemedicine for diabetes care in India during COVID19 pandemic and national lockdown period: guidelines for physicians. Diabetes Metab Syndr. 2020, 14:273-6. 10.1016/j.dsx.2020.04.001

14. Vaishya R, Bahl S, Singh RP: Letter to the editor in response to: Telemedicine for diabetes care in India during COVID19 pandemic and national lockdown period: Guidelines for physicians. Diabetes Metab Syndr. 2020, 14:687-8. 10.1016/j.dsx.2020.05.027

15. Prasad A, Brewster R, Newman JG, Rajasekaran K: Optimizing your telemedicine visit during the COVID-19 pandemic: practice guidelines for patients with head and neck cancer. Head Neck. 2020, 42:1317-21. 10.1002/hed.26197

16. Boehm K, Ziewers S, Brandt MP, et al.: Telemedicine online visits in urology during the COVID-19 pandemic-potential, risk factors, and patients' perspective. Eur Urol. 2020, 78:16-20. 10.1016/j.eururo.2020.04.055

17. Cinar P, Kubal T, Freifeld A, et al.: Safety at the time of the COVID-19 pandemic: how to keep our oncology patients and healthcare workers safe. J Natl Compr Canc Netw. 2020, 18:1-6. 10.6004/jnccn.2020.7572

18. Hodgkins M, Barron M, Jevaji S, Lloyd S: Physician requirements for adoption of telehealth following the SARS-CoV-2 pandemic. NPJ Digit Med. 2021, 4:19. 10.1038/s41746-021-00390-y 